\title{
L'architecture du pouvoir : projets et réalisations de palais du Parlement, de Dublin à Westminster
}

The Architecture of Power: Projects and Achievements in the Dublin and London Parliament Houses

Jacques Carré

\section{(2) OpenEdition}

\section{Journals}

Édition électronique

URL : http://journals.openedition.org/rfcb/1630

DOI : $10.4000 /$ rfcb. 1630

ISSN : 2429-4373

Éditeur

CRECIB - Centre de recherche et d'études en civilisation britannique

Édition imprimée

Date de publication : 1 janvier 2006

ISSN : 0248-9015

\section{Référence électronique}

Jacques Carré, «L'architecture du pouvoir : projets et réalisations de palais du Parlement, de Dublin à Westminster », Revue Française de Civilisation Britannique [En ligne], XIII-4 | 2006, mis en ligne le 01 janvier 2006, consulté le 20 avril 2019. URL : http://journals.openedition.org/rfcb/1630 ; DOI : $10.4000 /$ rfcb. 1630

Ce document a été généré automatiquement le 20 avril 2019

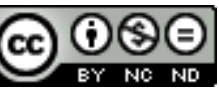

Revue française de civilisation britannique est mis à disposition selon les termes de la licence Creative Commons Attribution - Pas d'Utilisation Commerciale - Pas de Modification 4.0 International. 


\section{L'architecture du pouvoir : projets et réalisations de palais du Parlement, de Dublin à Westminster}

The Architecture of Power : Projects and Achievements in the Dublin and London Parliament Houses

Jacques Carré

1 Les édifices abritant les parlements nationaux ont une forte charge symbolique parce qu'ils abritent les représentants du peuple et sont associés à l'imaginaire national. Cette dimension symbolique a été confortée en Angleterre par la Glorieuse révolution de 1688, qui a institué une monarchie parlementaire équilibrant (en principe) les pouvoirs entre le roi, les Lords et les Communes. Le palais du Parlement, plutôt que le palais royal, fut désormais le lieu où s'exerçait et où se donnait à voir le pouvoir politique réel en GrandeBretagne. Le monarque y paraissait de temps en temps, notamment lorsqu'il y lisait chaque année le discours du trône, mais la chambre des Lords et celle des Communes devinrent les espaces privilégiés de la souveraineté nationale. On a plusieurs témoignages du début du XVIII e siècle sur l'importance nouvelle accordée à l'édifice abritant le Parlement : ceux de Defoe ${ }^{1}$, de Berkeley ${ }^{2}$ et de Shaftesbury ${ }^{3}$, notamment. Ces témoignages suggèrent que les contemporains étaient bien conscients de la symbolique architecturale du Parlement.

2 Il y avait en outre à cette époque des raisons pratiques de souhaiter la reconstruction du palais de Westminster. Les députés des Communes et les Lords étaient en effet très mal logés dans des salles anciennes, exiguës, mal aérées et mal chauffées. J. Ralph écrit en 1734 :

'Tis certain nothing can be more unworthy of so august a body as the parliament of Great Britain, than the present place of their assembly: it must be undoubtedly a great surprize to a foreigner, to be forc' $d$ to enquire for the Parliament-House even at the doors; and when he found it, to see it so detach'd in parcels, so incumber'd with wretched apartments, and so contemptible in the whole. ${ }^{4}$ 
Dans ces conditions on ne s'étonne pas que tout au long du XVIII siècle se soient succédé des projets de reconstruction du Parlement de Westminster. Pourtant, cette entreprise longtemps espérée ne fut décidée qu'après le grand incendie de 1834 , et c'est paradoxalement Dublin qui se vit dotée d'un nouvel édifice parlementaire, certes plus modeste, vers 1730, c'est-à-dire plus d'un siècle avant la capitale britannique. Notre étude portera donc autant sur des projets que sur des réalisations. La succession des projets de Parlement au XVIII ${ }^{\mathrm{e}}$ siècle présente en effet l'intérêt de refléter par leurs caractéristiques stylistiques un imaginaire politique nouveau et évolutif par rapport à l'avant-1688. Et c'est bien sûr ce lien entre esthétique et imaginaire national qui nous intéresse dans le cadre d'une réflexion sur art et nation.

\section{De la Rome antique aux îles Britanniques modernes}

Deux styles très différents ont caractérisé ces projets et réalisations d'édifices parlementaires: le style classique, dans ses différentes déclinaisons, du baroque au palladien et au néo-classique, d'une part; et le style néo-gothique, lui aussi divers dans ses formes. Jusqu'aux années 1790, le classique a dominé; ensuite, le gothique a connu une plus grande faveur, triomphant finalement en 1834, lorsque le palais de Westminster fut reconstruit après son incendie. Or, comme on le verra, le style classique dominant au XVIII siècle pouvait être qualifié de style cosmopolite, n'ayant rien de spécifiquement britannique. Une des questions qui seront donc posées ici est celle de la relation potentiellement conflictuelle entre un néo-classicisme architectural inspiré de l'Antiquité, d'une part, et les concepts de britannicité et d'irlandité, d'autre part. La fin du siècle, justement, ressuscitera le gothique au nom d'un certain nationalisme. L'épreuve des guerres révolutionnaires et napoléoniennes renforcera ce sentiment, et c'est sans doute pourquoi le concours ouvert pour la reconstruction du parlement incendié en 1834 exigera des architectes des projets en style gothique. Au-delà de l'opposition formelle entre un classicisme importé et un gothique censé être vernaculaire, on se demandera donc en quoi ce changement stylistique a contribué à exprimer l'évolution de l'idée de nation.

5 C'est bien sûr à la demande du Parlement lui-même, et notamment des Communes, aussi bien irlandaises que britanniques, que des projets de reconstruction furent préparés. Durant tout le XVIII e siècle l'office of Works, le Bureau des bâtiments royaux, était chargé d'entretenir les édifices nationaux, dans les deux pays. Mais cela ne veut pas dire que les plans de reconstruction émanèrent d'obscurs fonctionnaires. Des plans furent proposés par des architectes célèbres en leur temps, comme Nicholas Hawksmoor, Edward Lovett Pearce, Lord Burlington, William Kent, John Soane et James Wyatt. Mais on sait que la profession d'architecte telle que nous la connaissons maintenant ne fut définie qu'au début du XIX siècle, et qu'auparavant des catégories très diverses de personnes se livrèrent à l'exercice du dessin d'architecture. On peut justement remarquer qu'à Londres comme à Dublin les projets des années 1730 émanèrent d'amateurs d'architecture épris de culture classique et appartenant aux élites politiques: Lord Burlington, pair héréditaire, était membre de la chambre des Lords, et Edward Lovett Pearce était député aux Communes d'Irlande, et ami du Speaker des Communes William Conolly. Le premier était assisté de William Kent et d'autres dessinateurs membres de l'Office of Works; le second parvint à supplanter le Surintendant des bâtiments royaux en Irlande, Thomas Burgh, en faisant valoir sa vaste culture architecturale. Ni Kent, ni Burgh, sans appui, 
n'auraient jugé utile de proposer de tels plans. L'éminence sociale était à cette époque une condition indispensable pour espérer faire aboutir un projet de bâtiment public de cette importance. En même temps, le fait que les concepteurs aient été membres de l'oligarchie dirigeante conférait une sorte de légitimité politique à leurs projets.

Nous verrons que cette légitimité trouva sa traduction architecturale dans un vocabulaire architectural classique, inspiré très largement de l'Antiquité et de la Renaissance italienne, avec usage des ordres, recours à des proportions mathématiques simples, vocabulaire décoratif codifié, et, bien sûr, imitation de certains types d'édifices antiques comme les temples, les basiliques ou les thermes. En nous fondant sur une réalisation, celle du Parlement de Dublin, et sur un projet pour le Parlement de Westminster datant des années 1730, au moment de l'essor du palladianisme, nous tenterons d'expliquer pourquoi, dans les deux capitales, ce style fut choisi par les promoteurs de nouveaux édifices parlementaires.

\section{Le nouveau Parlement de Dublin}

7 En 1728 Edward Lovett Pearce (vers 1697-1733), fils d'un général irlandais, député aux Communes d'Irlande depuis 1726 et architecte amateur, proposa le plan d'un nouveau Parlement ${ }^{5}$. Normalement les édifices publics irlandais étaient du ressort du Surintendant des bâtiments royaux, Thomas Burgh. Mais Pearce mit en avant ses compétences particulières, sa connaissance de l'Italie et des grands traités d'architecture, et laissa entendre qu'un bâtiment aussi exceptionnel ne pouvait pas être confié à un tâcheron comme Burgh (surtout connu pour la réalisation à Dublin d'un hôpital et d'une caserne). Pearce avait en outre le soutien du Speaker de la chambre des Communes irlandaise, William Conolly, ce qui était évidemment un appui majeur.

8 Nous avons la chance de pouvoir connaître les intentions de l'architecte grâce à un mémorandum de 1728 adressé à une commission parlementaire ${ }^{6}$. Pearce proposa un seul plan, mais deux versions de la façade du Parlement, l'une meilleur marché que l'autre. La plus coûteuse comportait deux arcs de triomphe symétriques, de part et d'autre d'un corps central. On avait en effet demandé deux entrées séparées à l'architecte, l'une pour les Communes, l'autre pour les Lords. Ce choix du motif de l'arc de triomphe était un rappel typiquement historiciste de la Rome antique, réutilisant à des fins purement décoratives un édifice urbain normalement isolé. La version moins chère, qui fut réalisée, ne comprenait qu'une baie surmontée d'un fronton triangulaire à la place de l'arc de triomphe. Les deux entrées furent réunies par un péristyle construit, selon l'architecte « à la manière du péristyle grec décrit par Vitruve ». Pearce indique également dans son mémorandum la proportion exacte des différentes salles, suivant en cela l'exemple de Palladio et Jones, entre autres.

De manière plus originale, le plan du nouveau Parlement (figure 1) installe la chambre des Communes au centre de l'édifice, la chambre des Lords étant placée sur le côté, comme une sorte d'appendice. Ce choix a été interprété en termes politiques par E. McParland comme le signe d'une mise en avant symbolique de l'importance des Communes, et d'une minoration du rôle des Lords. Cette interprétation paraît crédible, dans la mesure où l'opinion publique irlandaise avait récemment manifesté de l'hostilité aux décisions du gouvernement de Londres, notamment lorsque le Declaratory Act de 1720 avait tenté d'imposer la subordination des Communes irlandaises au Parlement de Westminster. Et l'on sait la réaction violente qui avait accueilli la décision de Londres de mettre en 
circulation en Irlande des pièces d'un demi-penny en bronze (les fameux Wood's half-pence dénoncés par Swift).

La chambre des Communes, en outre, ne rappelait en rien la chapelle de St Stephen, où se tenaient les sessions des Communes de Westminster. Le plan octogonal choisi permit d'installer des sièges tout autour de la salle, ce qui changeait de la séparation bien tranchée entre les partis sur les bancs de St Stephen's. L'architecte invoqua des raisons acoustiques et disciplinaires à cette disposition :

I have endeavourd by this Figure to give all Advantages necessary for such an Assembly, The Chair is free at very near an equal Distance from all Parties, the Speaker can from thence with very little Difficulty see every Body in their seats, and every person who speaks from his seat so as to be heard by the speaker must be heard by every body. The Barr has also the same Advantages, and an Evidence in the Center, must be heard equally by every one. Order and Attention is with great Difficulty preserved where Gentlemen can talk to each other cross the Floor, or are seated in Clusters especially behind the Chair, which cannot possibly happen in this situation. $^{7}$

11 C'était encore une fois mettre en avant le souci d'efficacité de l'institution. Ajoutons qu'une spacieuse galerie des visiteurs était installée au premier étage, entourant complètement la Chambre, et capable d'accueillir près de 700 personnes, alors qu'il y avait seulement 280 sièges pour les députés. Cette galerie fait ressembler la chambre des Communes à un théâtre ${ }^{8}$, et peut-être faut-il voir dans cette disposition une mise en valeur de l'importance de l'opinion publique dans la vie politique irlandaise.

Figure 1 : Plan du Parlement de Dublin au XVIII e siècle (gravure de R. Omer, 1767)

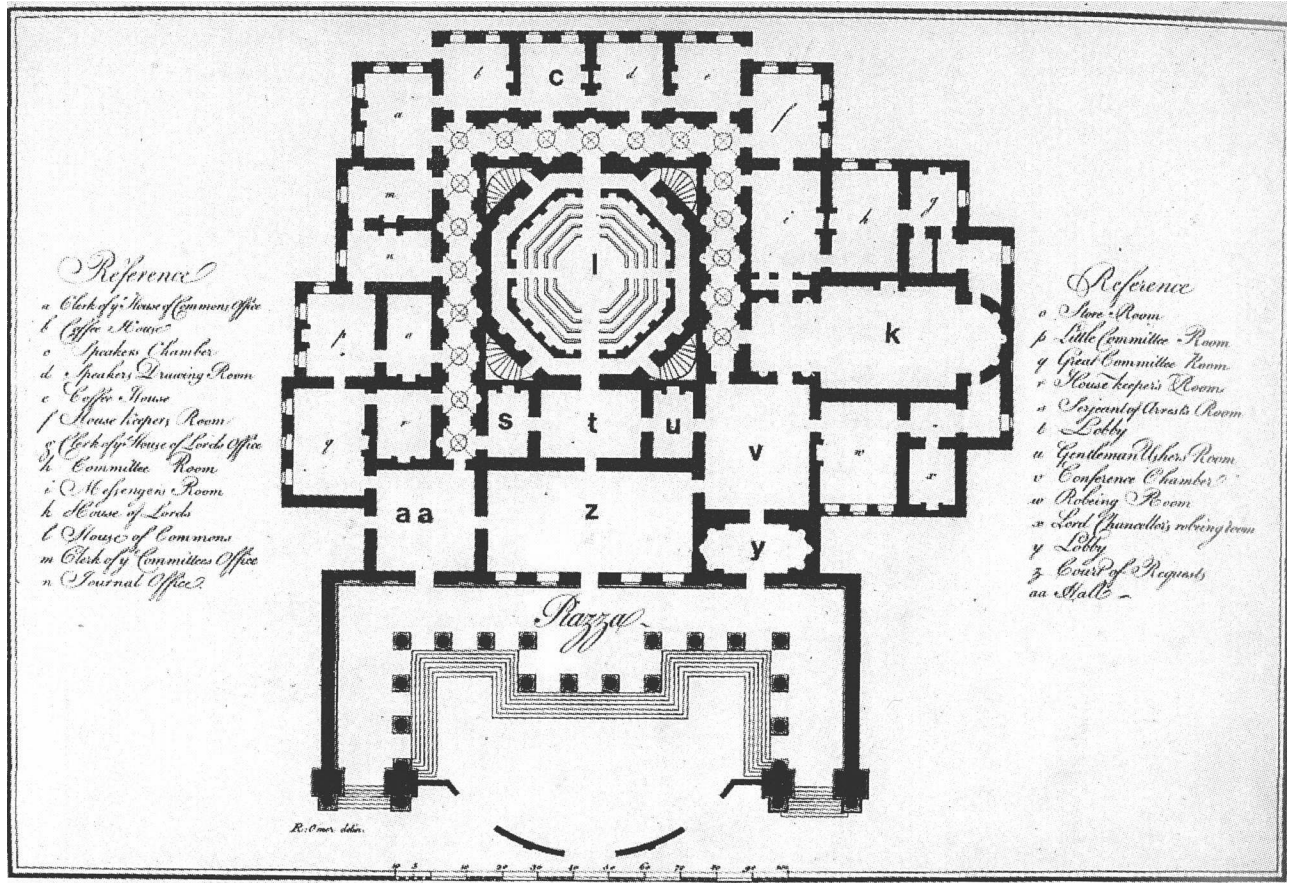

12 Formant contraste avec la chambre des Communes, la chambre des Lords d'Irlande, qui seule subsiste de nos jours, était beaucoup plus petite, étant prévue pour permettre à seulement trente présents de s'asseoir (on n'attendait plus les absentees!). Sa décoration devait beaucoup à l'architecture antique. Pearce expliqua qu'elle associait «l'exèdre grecque mentionnée par Vitruve à la salle corinthienne de Palladio ». En même temps, il 
recommandait que du marbre de Kilkenny, donc provenant du royaume d'Irlande, fût choisi pour les colonnes. L'adhésion explicite de Pearce au palladianisme, tout à fait nouvelle en Irlande, suggère à quel point il était soucieux de manifester sa vaste culture architecturale, et de tourner le dos au particularisme architectural. Il inscrivit son édifice dans une tradition cosmopolite, mais en même temps il proposa aussi bien pour les Communes que pour les Lords, des plans adaptés à la situation politique irlandaise. Nous découvrons donc ici une tension entre universalisme et particularisme qui est, je crois, un aspect important de la problématique art et nation dans les îles Britanniques.

\section{Les projets de nouveau Parlement à Westminster au $\mathrm{XVIII}$ e siècle}

Le deuxième édifice qui me paraît significatif d'une volonté de renaissance à la fois artistique et nationale est le projet de reconstruction du palais de Westminster élaboré à partir de 1732, peu après l'achèvement du Parlement de Dublin, par les architectes du cercle de Lord Burlington (1694-1753) ${ }^{9}$. Ce dernier, pair d'Angleterre et architecte amateur, avait déjà rassemblé une équipe d'architectes autour de lui, avec notamment William Kent (1685-1748), dans le but de diffuser en Angleterre le palladianisme auquel il vouait un culte. Il est même possible qu'il ait déployé tant d'efforts pour placer ses protégés à l'Office of Works afin de faciliter la réalisation d'un Parlement néo-palladien. On ne sait malheureusement pas si la réalisation du nouveau Parlement de Dublin eut une influence quelconque sur le projet londonien, bien que leurs styles aient été fort semblables.

Une commission de la Chambre des Communes fut réunie en 1732 afin de déterminer le site d'un éventuel nouvel édifice pour abriter le Parlement. Hawksmoor, en tant que secrétaire du conseil de l'office of Works, fournit cette année-là des plans (aujourd'hui disparus) qui furent soumis en mars à l'examen de Burlington. On peut supposer que les propositions de Hawksmoor ne recueillirent pas son approbation, car il mit sa propre équipe d'architectes au travail, comme on l'apprend par le Gentleman's Magazine du mois de mars 1733 : 'The Earl of Burlington has projected a Plan for building two new Houses of Parliament, and a Public Library between them, to be finish'd against next session, and to cost the Public about 30,000 pounds.' Ce texte, confirmé par un autre article de presse contemporain, fournit la preuve formelle que le promoteur des projets présentés à partir de 1733 fut bien l'aristocrate, même si tous les dessins qui subsistent sont de la main de William Kent ou d'autres architectes de l'office of Works.

Les différents projets présentés, aussi bien en 1733 qu'en 1739, présentent un bâtiment rectangulaire, tantôt de 150 mètres de long, tantôt réduit à 100 mètres seulement, et abritant les deux chambres ainsi que la Bibliothèque Cottonienne. Il devait être situé au sud de Westminster Hall, la vénérable salle médiévale, qui était conservée. Il est à noter qu'aucun des projets de Parlement du XVIII ${ }^{e}$ siècle ne fit table rase de cette salle, bien que son style ne s'accordât pas du tout avec le classicisme des projets. La capitale britannique n'était pas encore conçue en termes de paysage urbain, et chaque édifice public était considéré comme une entité autonome affirmant son message propre. Comme à Dublin, le Parlement proposé par le cercle de Burlington s'inspirait explicitement de modèles antiques. L'abondance de dessins qui subsistent se caractérise par une grande cohérence stylistique. 

dont les façades principales devaient donner, à l'Ouest, sur Old Palace Yard et à l'Est sur la Tamise. Le centre de la façade occidentale devait comporter une colonnade corinthienne géante reposant sur un socle en appareil rustique. Un portique hexastyle s'en détachait au milieu, surmonté par un dôme surbaissé de type thermal. Chacun des corps latéraux comportait deux tours carrées en saillie.

Un autre projet, daté de 1739 (figure 2), montre un édifice plus compact, assez semblable dans son décor extérieur, et dont le plan révèle encore plus nettement l'influence directe de l'architecture des thermes antiques. Si les deux chambres, disposées symétriquement, ont un plan rectangulaire fort simple, les autres salles sont remarquables par la variété de leurs formes basilicales ou ovoïdes. Il faut dire que Lord Burlington venait justement de faire préparer une édition des dessins de Palladio représentant des thermes antiques, Fabbriche Antiche Disegnate da Andrea Palladio Vicentino ${ }^{10}$. Ce volume fournit tout le vocabulaire architectural des projets de Parlement de son équipe : à l'extérieur, fenêtres "dioclétiennes", dômes surbaissés, colonnades et portiques ; à l'intérieur, portiques libres, voûtes à caissons et succession de salles aux formes systématiquement différentes.

Figure 2 : Plan projeté pour le Parlement de Westminster (dessin de W. Kent, 1739)

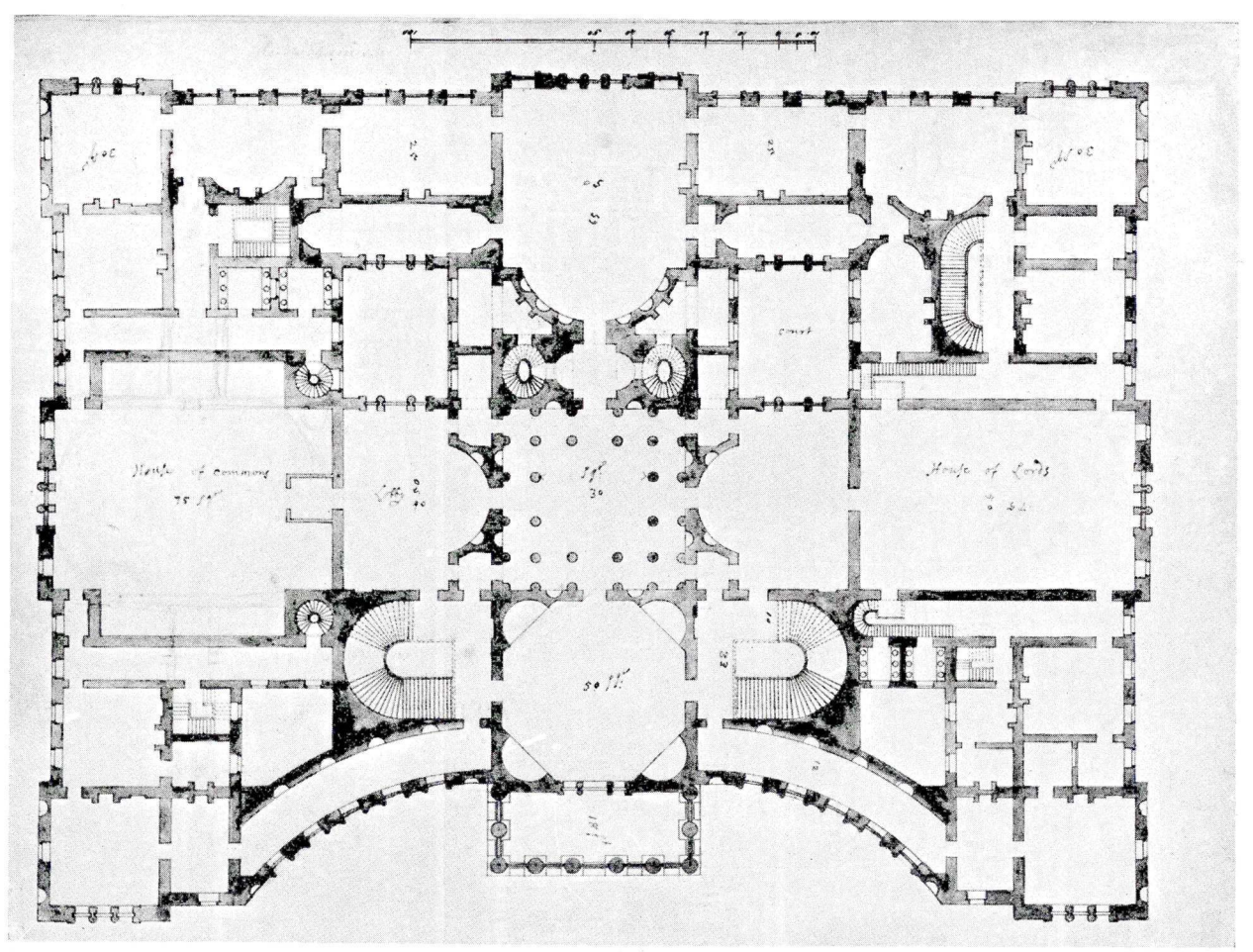

Le caractère assez grandiose, voire théâtral, de ces projets avait de quoi effrayer les fonctionnaires du Trésor et les ministres par son ampleur et son coût imprévisible qui aurait assurément dépassé très largement les trente mille livres mentionnées dans le Gentleman's Magazine en mars 1733. En 1739, la chambre des Communes manifesta de nouveau son intention d'obtenir la construction d'un nouveau palais du Parlement. Mais ce fut de nouveau peine perdue pour les architectes, car la guerre contre l'Espagne accapara bientôt l'attention des gouvernants et interdit les dépenses somptuaires. Ainsi fut définitivement enterrée la plus ambitieuse entreprise jamais conçue dans le cercle de Burlington. 


\section{Les caractéristiques de l'anglo-palladianisme} Grande-Bretagne était parfaitement accordée à la mythologie politique des whigs, qui invoquait volontiers la République romaine et son équilibre des pouvoirs comme précédent illustre au régime mixte issu de 1688. La philosophie politique du whiggisme s'appuyait largement sur la lecture de l'Antiquité par Polybe, Machiavel et Harrington, en même temps qu'elle se nourrissait des théories de Locke. Un Parlement siégeant dans une architecture à l'antique pouvait se targuer d'être logé dans le décor architectural le plus approprié. Cette harmonie entre les principes politiques et le décor architectural n'avait rien pour déplaire à l'oligarchie whig, qui présentait volontiers le régime parlementaire issu de 1688 comme un modèle de régime éclairé.

\section{Gothique et britannicité}

Ce désir de forger une image nouvelle, une image moderne du pays, aussi bien dans les domaines intellectuel et artistique que politique, impliquait évidemment des emprunts à l'art et à la civilisation européens, et pouvait être perçu comme un manque de loyauté par rapport à la nation. Tout un discours satirique xénophobe se développa au XVIII ${ }^{e}$ siècle, 
on le sait, à propos des formes artistiques importées d'Italie, qu'il s'agisse de musique, de peinture, ou d'architecture. Un sentiment de fierté patriotique s'exprima à partir du milieu du siècle chez certains artistes comme William Hogarth et Francis Hayman.

L'exploitation de la fibre patriotique, toutefois, n'était pas toujours innocente, et prenait souvent sa source dans l'opposition politique, qu'il s'agisse de factions opposées à Walpole, ou, plus tard, des Tories ou des Radicaux. Par exemple, parmi les sources du whiggisme volontiers invoquées par l'opposition à Walpole vers 1730, on trouvait des mythes politiques nordiques, associés à la Germanie barbare, telle que Tacite l'avait décrite, ainsi qu'aux envahisseurs saxons de la Grande-Bretagne, censés avoir été les défenseurs des libertés individuelles. Le gothique de fantaisie du XVIII siècle, dénommé Gothick, fut consciemment associé au mythe des libertés saxonnes, par exemple dans le jardin de Stowe, par Lord Temple, opposant à Walpole, et ceci dès 1741, avec le « Temple de la Liberté » dessiné par James Gibbs.

Mais ce gothique était aussi évidemment apprécié pour des raisons opposées à celles qui justifiaient le classicisme. Il était aimé parce qu'il n'était ni savant, ni moderne, ni universellement intelligible, mais justement parce qu'il paraissait laisser libre cours à l'imagination individuelle. Comme l'a montré Michel Baridon, il était mieux accordé que le style classique à l'épistémologie nouvelle, notamment à la psychologie associationniste ${ }^{11}$. En outre, il était présenté comme ayant ses racines dans l'histoire nationale. Dans le contexte des nombreuses guerres avec la France de la deuxième moitié du siècle, cet argument ne pouvait manquer de flatter le patriotisme naissant. Certains antiquaires comme John Carter déclarèrent que le Gothique était né en Angleterre ${ }^{12}$. Dans le contexte anti-français de la fin du XVIII siècle, ce genre d'architecture avait donc toutes les chances de réunir les suffrages d'une partie de l'opinion, moins pour des raisons esthétiques que patriotiques. Il est clair que durant les guerres révolutionnaires et napoléoniennes, les activités artistiques de toute sorte furent souvent l'occasion d'exprimer le sentiment patriotique.

Et l'on ne peut donc s'étonner que dès cette époque, de manière d'abord discrète, le néoGothique soit apparu à Westminster. Les premières escarmouches entre les tenants des deux styles eurent lieu dès la fin du siècle. Ce fut à l'occasion d'une lutte d'influence entre John Soane, tenant d'un néo-classicisme fort original, et James Wyatt, amateur de gothique pittoresque. En 1794, Soane et son atelier produisirent plus de 270 dessins détaillant un nouveau parlement néo-classique. Mais l'heure n'était pas propice à des dépenses aussi importantes. Et ce fut James Wyatt, qui, en 1799, fut chargé d'aménager le vieux palais à l'occasion de l'union politique avec l'Irlande et de l'arrivée prochaine de parlementaires plus nombreux à Westminster. Dans une partie de l'édifice existant, il créa consciemment un décor urbain pittoresque, avec une façade néo-gothique donnant sur Old Palace Yard, destinée à harmoniser de vieux bâtiments disparates abritant notamment la chambre des Lords. John Carter, ennemi juré du gothique de fantaisie, cria au scandale parce que les matériaux étaient peu nobles (Wyatt utilisa surtout brique, stuc et ciment) et que cette façade s'apparentait à un décor de théâtre :

As the bricklayers elevate themselves, the Compoists follow, hand to fist, trowel to trowel, rubbish to rubbish. And thus the work is perfected; smeared over at the same time with the Housepainter's brush, by way of pencilling mock masonry joints, and mock tints of perforated stone-work. ${ }^{13}$ grandissant du gothique chez les élites à la fin du XVIII ${ }^{e}$ siècle. Le triomphe du gothique 
fut conforté après l'incendie de 1834, qui détruisit les deux chambres et leurs alentours, épargnant seulement Westminster Hall. Désormais, une reconstruction complète du Parlement était nécessaire, et le gouvernement n'avait plus le choix ${ }^{14}$. La commission parlementaire chargée de l'affaire décida en juin 1835 d'organiser un concours architectural. Cette décision était historique, puisque jusqu'ici, comme nous l'avons vu, les jeux d'influence avaient été décisifs dans la sélection des projets. Un concours ouvert à tous les architectes professionnels supposait que l'apparence du Parlement devenait une affaire publique, et ne dépendait plus (du moins en théorie) de l'influence de tel ou tel membre des élites.

La deuxième décision importante de la commission parlementaire de 1835 fut le choix du style architectural du nouveau Parlement. On aurait pu penser qu'après un siècle de projets classiques, et au moment où le néo-classicisme triomphait sur le continent, la commission aurait recommandé le classicisme, ou du moins n'aurait pas imposé de style. Et pourtant elle recommanda que le style du nouvel édifice soit «ou gothique ou élisabéthain $»^{15}$. La justification explicite de ce choix était que l'apparence du nouveau Parlement devait s'harmoniser avec la cathédrale de Westminster et avec Westminster Hall. Mais d'autres raisons du choix du gothique furent explicitées durant la vive controverse qui se déclencha alors entre les tenants du classicisme et ceux du gothique. Le plus habile défenseur du classicisme fut le diplomate William Richard Hamilton, qui publia en 1836 deux Lettres à Lord Elgin dans lesquelles il affirma que « le seul mot d'édifice gothique est synonyme de l'idée la plus débridée et la plus fantasque que n'importe quel artiste ou amateur peut concevoir à sa guise $»^{16}$. Les défenseurs du gothique, comme par exemple le colonel Jackson, invoquèrent en revanche le caractère national de ce style, sa beauté pittoresque, et son lien avec l'histoire de l'institution parlementaire ${ }^{17}$.

La troisième décision de la commission fut que la nouvelle chambre des Communes devait pouvoir accueillir la totalité de ses membres, soit 658 députés. Ceci était une petite révolution par rapport à la chapelle de St Stephen, exiguë, peu confortable et notoirement incapable d'offrir un siège à tous les députés. Cette décision est bien caractéristique de l'esprit de réforme qui souffla sur le royaume dans les années 1830 : l'électorat, récemment doublé par la loi électorale de 1832, allait être mieux représenté, dans tous les sens du terme, à la chambre des Communes. La nouvelle représentativité du Parlement allait pouvoir être donnée en spectacle aux visiteurs. 

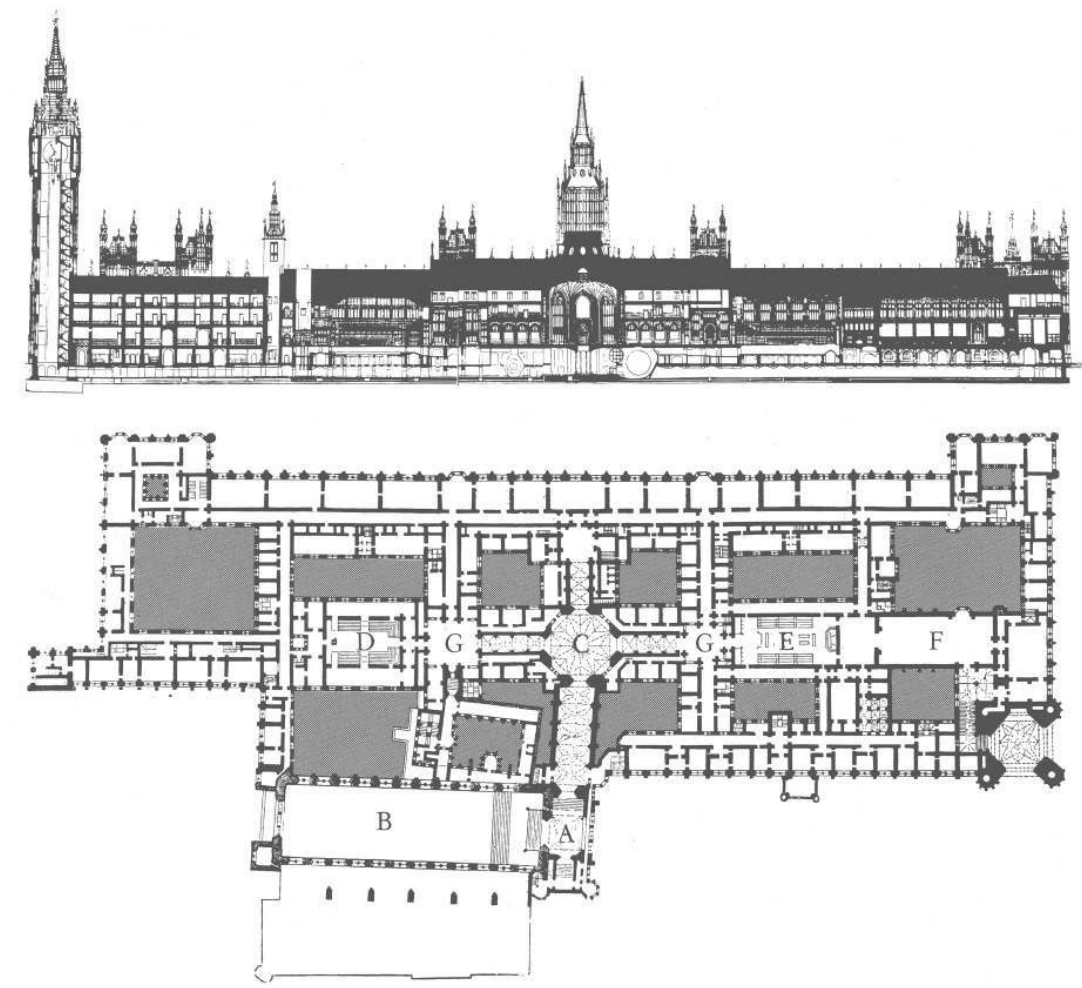

Le caractère public de la reconstruction du Parlement fut renforcé par l'organisation au printemps 1836 d'une exposition où furent présentés les 97 projets envoyés par les participants au concours. Quatre d'entre eux, toutefois, avaient déjà été pré-sélectionnés par la commission, et celui de Charles Barry (1795-1860) placé en tête et recommandé à l'approbation du souverain (figure 3). Ce fut l'heureux vainqueur qui durant de très longues années (l'édifice ne fut achevé que vers 1860) dirigea la construction de l'édifice que nous connaissons, en confiant la riche décoration intérieure à Augustus Welby Pugin (1812-1852), autre gothiciste convaincu.

31 Le nouveau palais de Westminster s'ancra ainsi de plusieurs manières dans l'imaginaire national. Loin d'affirmer une modernité cosmopolite, comme les projets palladiens et néo-classiques du siècle précédent, il s'inscrivait dans une continuité historique plus ou moins réinventée. D'abord, il englobait le vieux Westminster Hall, désormais promu lieu de mémoire, et s'harmonisait avec la cathédrale de Westminster, pour former un ensemble asymétrique d'un pittoresque bien anglais. On peut trouver dans cette homogénéité stylistique entre le nouveau Parlement et les monuments voisins le désir de traduire symboliquement une continuité politique avec le Moyen-Âge, souvent évoqué comme le moment de l'essor de l'institution. D'autre part, l'inspiration ecclésiastique du Parlement de Barry, avec ses deux hautes tours proches de celles de la cathédrale de Westminster, pouvait aussi rappeler l'alliance de l'Église et de l'État, à une époque où elle paraissait menacée par la tolérance accordée aux dissidents et aux catholiques par des lois récentes. Ainsi, le nouveau palais de Westminster pouvait être perçu comme une célébration d'un passé dont les Britanniques étaient fiers.

Mais on peut aussi avancer que son extrême originalité pour son temps représentait un défi au goût dominant dans les autres pays. Le Parlement gothique de Barry revendiquait 
hautement un particularisme national, à une époque où le néo-classicisme international triomphait encore sur le Continent européen et aux États-Unis. N'était-ce pas une manière d'affirmer que le Royaume-Uni avait enfin acquis son autonomie dans le domaine artistique, au moment où il devenait la première puissance mondiale?

\section{Conclusion}

S'il est imprudent de relier de manière trop rigide l'évolution diversifiée de la création artistique avec celle de la société d'une époque donnée, on peut néanmoins suggérer que l'éclipse du classicisme architectural si longtemps dominant au XVIII ${ }^{\mathrm{e}}$ siècle a eu quelque chose à voir avec la lente mutation sociale et politique qui a vu l'ascension de nouvelles élites issues du monde du commerce et de l'industrie. Le culte de l'Antiquité classique si évident dans les projets palladiens de Parlement était perçu à la fin du XVIII ${ }^{\mathrm{e}}$ siècle comme l'apanage des élites foncières traditionnelles, comme un langage visuel codifié qui lui était spécifique. Mais les caractéristiques du classicisme savant, moderne et universel du XVIII ${ }^{e}$ siècle n'avaient plus un siècle plus tard le même attrait, pour une opinion britannique désormais hostile au cosmopolitisme aristocratique et puisant son identité dans un protestantisme éminemment national. Le gothique, avec sa malléabilité et son absence de règles, avec son ancrage dans l'histoire et le territoire anglais, pouvait au contraire apparaitre comme un langage plus adapté à une période de mutation politique dont la réforme électorale de 1832 est un exemple symbolique. On peut suggérer, me semble-t-il, que le triomphe du gothique à Westminster, avec le choix du projet de Parlement de Barry en 1836, et l'admiration exprimée par l'opinion publique une fois le bâtiment construit, ne prend tout son sens que dans son rapport avec la redéfinition des élites du pays. Associer l'institution parlementaire au passé national par l'intermédiaire de l'architecture gothique, n'était-ce pas pour les membres des élites traditionnelles des années 1830-40 un moyen de se relégitimer en modifiant leur ancienne image de marque cosmopolite? En même temps, c'était sans doute un moyen de flatter les tendances profondes des classes moyennes, étrangères à la culture classique et sensibles au discours identitaire que développaient les gothicistes.

\section{NOTES}

1. 'The House of Lords is a venerable old place, indeed ; but how mean, how incoherent, and how straitened are the several avenues to it, and rooms about it? The matted gallery, the lobby, the back ways the King goes to it, how short are they all of the dignity of the place, and the glory of a King of Great Britain, with the Lords and Commons, that so often meet there?' in Daniel DEFOE, A Tour through the Whole Island of Great Britain (1724), ed. P. Rogers, Harmondsworth: Penguin Books, p. 324.

2. George BERKELEY, An Essay towards preventing the Ruine of Great Britain, London, 1721, p. 19.

3. Anthony Ashley Cooper, $3^{\text {rd }}$ Earl of SHAFTESBURY, 'A Letter Concerning Design' (1712) in Second Characters, or the Language of Forms, ed. B. RAND, Cambridge: University Press, 1914, p. 21. 
4. James RALPH, A Critical Review of the Publick Buildings, Statues and Ornaments in and about London and Westminster, London, 1734, pp. 56-57.

5. Pour une étude détaillée de la construction de cet édifice, on se reportera aux travaux d'Edward McPARLAND : 'Edward Lovett Pearce and the Parliament House in Dublin', Burlington Magazine, vol. CXXXI, n¹031, février 1989, pp.91-100 et 'Building the Parliament House in Dublin', Parliamentary History, vol. 21 (2001), pp. 131-140; voir aussi le chapitre 7 de son ouvrage Public Architecture in Ireland, 1680-1760, Newhaven \& London: Yale University Press, 2001.

6. Ce mémorandum est reproduit in McPARLAND, loc. cit. (1989), p. 100.

7. Ibid.

8. C'est du moins l'impression que donne le tableau de Francis WHEATLEY, The Irish House of Commons (1780), Lotherton Hall, Leeds.

9. On peut encore se reporter à l'étude ancienne détaillée de Fiske KIMBALL, 'William Kent's Designs for the Houses of Parliament', R.I.B.A. Journal, vol. XXXIX, août 1932, pp. 733-755 et septembre 1932, pp. 801-807. Des dessins plus récemment retrouvés sont pris en compte par Howard Colvin, The History of the King's Works, V, 1660-1782, London: H.M.S.O., 1976, pp. 416-425.

10. Ce volume daté de 1730 fut en réalité publié quelques années plus tard, en tirage extrêmement limité.

11. Michel BARIDON, «Forme vivante et forme mathématique : la dynamique de l'imaginaire gothique en Angleterre ", in Le Jardin paysager anglais au dix-huitième siècle, Dijon: Éditions universitaires de Dijon, 2000, pp. 23-40.

12. John CARTER, The Ancient Architecture of England, including the Orders during the British, Roman, Saxon, and Norman Eras, London, 1795, préface.

13. Cité par H.M. COLVIN ed., The History of the King's Works, VI, 1782-1851, London: H.M.S.O., 1973., p. 518.

14. Sur la construction du nouveau palais de Westminster, on pourra lire The Houses of Parliament : History, Art, Architecture, ed. C. \& J. RIDING, London: Merrell Publishers, 2000 ; ainsi que l'étude de M.H. PORT dans H.M. COLVIN, ed., op. cit., pp. 573-626.

15. COLVIN, op. cit., p. 576.

16. William R. HAMILTON, Second Letter to the Earl of Elgin, one of the Trustees of the British Museum, London, 1836, p. 75.

17. Alfred BARRY, The Life and Works of Charles Barry, London, 1867, p.151.

\section{RÉSUMÉS}

L'article étudie le lien entre l'évolution de l'architecture des palais du Parlement, de l'identité britannique et de l'image des élites politiques entre 1730 et 1840 . Vers 1730, le Parlement édifié à Dublin et les projets palladiens pour le Parlement de Londres montrent l'attrait du classicisme cosmopolite inspiré de l'Antiquité pour les élites whig. Le choix du style gothique lors de la reconstruction du Parlement de Londres après 1834 traduit au contraire une identification des élites avec le génie national et l'accession du Royaume-Uni au rang de pays artistiquement autonome.

The paper examines the link between the way in which the architecture of Parliament Houses, British identity and the image of political elites evolved from 1730 to 1840 . Towards 1730 , the new Dublin Parliament and the Palladian projects in London illustrated the appeal of a 
cosmopolitan classicism inspired by Antiquity for the Whig élites. On the contrary, the choice of Gothic for the reconstruction of the London Parliament after 1834 expresses the identification of the élites with the national genius and the accession of the United Kingdom to autonomy in the field of the fine arts.

\section{AUTEUR}

\section{JACQUES CARRÉ}

Université Paris IV-Sorbonne 